\title{
DiSEÑO DE UNA EXPERIENCIA FLIPPED CLASSROOM Y TICS EN EL AULA
}

DESIGN OF A FLIPPED ClasSROOM EXPERIENCE AND ICT IN THE CLASSROOM

\author{
Macario Rodríguez-Entrena* \\ Nazaret M. Montilla-López \\ Carlos Gutiérrez-Martín \\ Manuela Castillo Quero \\ *mrentrena@uco.es \\ Universidad de Córdoba
}

Received: 30/06/2018

Accepted: 30/10/2018

\section{Resumen}

La metodología "Flipped Classroom" elimina el enfoque tradicional y lo reemplazan con tareas activas en clase y trabajo previo y posterior a la clase. A pesar de la popularidad de estos enfoques en los medios de comunicación y cursos impartidos a docentes relacionados con esta metodología, hay muy poca evidencia de la efectividad o consistencia en la comprensión de lo que en realidad sucede en las aulas. Este trabajo pretende analizar si el uso combinado de los recursos disponibles, webs temáticas y la edición de vídeos por parte del profesorado mediante el enfoque "Flipped Classroom" mejora el aprendizaje del alumno. Asímismo, se pretende evaluar si dicha innovación metodológica presenta un impacto diferencial en la evaluación final cuando se compara el método tradicional con dicha innovación docente, mediante el uso de análisis estadístico. Los resultados de la experiencia no han arrojado diferencias significativas, pero han sido positivos debido a que la metodologúa Flipped Classroom mejora el feedback que el profesorado recibe del alumnado en tiempo real, permitiendo adaptarse al ritmo de aprendizaje del alumnado. Sin embargo, el propio alumnado reconoce que no puede entenderse como una estrategia de sustitución del profesor. En este sentido, la implementación práctica del engfoque "Flipped Classroom" requiere necesariamente de una plasmación tácita en la guía docente para tener el poder vinculante necesario para que el estudiante haga el esfuerzo de revisar en casa el soporte audiovisual temático y los materiales de apoyo.

Palabras clave: Flipped Classroom, TICs, Motivación

\section{Abstract}

The "Flipped Classroom" methodology eliminates the traditional approach and replaces it with active tasks in class and study before and after class. Despite the popularity of these approaches in the media and courses given to teachers related to this methodology, there is very little evidence of the effectiveness or consistency in understanding what actually happens in the classroom. This paper aims to analyze whether the combined use of available resources, thematic websites and the video editing by teachers through the "Flipped Classroom" approach improves student learning. Likewise, it is intended to evaluate if such methodological innovation presents a differential impact in the final evaluation when this teaching innovation is compared with the traditional method through the use of statistical analysis. The results of the experience have not shown significant differences, but they have been positive because the Flipped Classroom methodology improves the feedback that the teachers receive from the students in real time, allowing them to adapt to the student's learning rhythm. However, the students themselves recognize that it can not be understood as a teacher substitution strategy. In this sense, the practical implementation of the "Flipped Classroom" approach necessarily requires its tacit inclusion in the teaching guide in order to have the necessary binding power for the student to make the effort to review the thematic audiovisual and additional support materials at home.

Keywords: Flipped Classroom, TICs, Motivation 


\section{INTRODUCCIÓN}

Los enfoques de Flipped Classroom han sido objeto de mucha atención desde el acuñamiento del término en torno a 2011, y su popularidad como término de búsqueda de Google (2013) ha aumentado exponencialmente (Abeysekera y Dawson, 2015; Albert y Beatty, 2015; O'Flaherty, J. y Phillips, 2015). Además, entre las múltiples opciones a la hora de aplicar nuevas tecnologías de la información y la comunicación (TIC) en el aula universitaria destacan las webs temáticas y la edición de vídeos por parte del profesorado, las cuales conforman algunas de las principales herramientas del denominado e-learning (Good y Lavigne, 2017). Con ambas herramientas se pretende dotar de gran accesibilidad a toda la información acerca del desarrollo y evaluación de la asignatura, los contenidos y actividades que se realizarán, materiales y recursos necesarios, bibliografía básica y complementaria. Mediante el proyecto de innovación docente realizado se ha pretendido mejorar el proceso de enseñanzaaprendizaje aplicando estos conceptos.

La experiencia docente acumulada, junto con los cambios en la organización docente a raíz de la implantación de los nuevos grados, impulsa la necesidad de mejorar la metodología en dos direcciones (Bishop y Verleger, 2013). En numerosos trabajos se ha señalado que los alumnos hacen un uso intensivo y continuo de las webs temáticas, de manera que en general acceden a las mismas a lo largo de todo el cuatrimestre y como apoyo a la docencia presencial. Sin embargo, no siempre acceden a los sitios webs apropiados y muchas veces la información no está completa, por lo que el profesorado debe orientar en este sentido. Adicionalmente, existen diversos factores que podrían complementar el uso de las mismas, tal y como la edición de recursos virtuales por parte del profesorado. Para ello, se ha propuesto aplicar el enfoque "Flipped Classroom" (aula invertida) donde se invierte el método de enseñanza tradicional de modo que el alumnado tiene su primer contacto con los contenidos de la asignatura fuera del aula. Básicamente, el concepto de una "Flipped Classroom" es el siguiente: lo que se hace tradicionalmente en clase ya está hecho en casa, y lo que tradicionalmente se hace como tarea se completa ahora en clase (Bergmann y Sams, 2012). Adicionalmente, durante las sesiones de "Flipped Classroom" se fomentará el trabajo colaborativo que ayuda a la mejor comprensión e interiorización de los contenidos (Estévez et al., 2018).

En el proyecto que se propone se pretende analizar si el uso combinado de los recursos mencionados mejora el aprendizaje del alumnado y la receptividad de los mismos ante esta metodología. Asímismo, se pretende evaluar si dicha innovación metodológica presenta un impacto diferencial en la evaluación final cuando se compara el método tradicional con dicha innovación docente. Esta experiencia piloto, basada en la metodología "Flipped Classroom" es totalmente idónea para integrar tanto la competencia CB1: conocimiento en materias básicas, científicas y tecnológicas que permitan un aprendizaje continuo, así como una capacidad de adaptación a nuevas situaciones o entornos cambiantes; CB3: capacidad de liderazgo, comunicación y transmisión de conocimientos, habilidades y destrezas en los ámbitos sociales de actuación y CB5: capacidad para desarrollar sus actividades, asumiendo un compromiso social, ético y ambiental en sintonía con la realidad del entorno humano y natural.

Por tanto, esta metodología va a ser implementada en diversas titulaciones: Doble Grado en Derecho y Administración y Dirección de Empresas, Máster de Ingeniería Agronómica, Grado en Ingeniería Agronómica y del Medio Rural, y Grado en Ingeniería Forestal. Las asignaturas en las cuales va a ser implementada esta experiencia piloto son: Investigación de Mercados e Investigación de Mercados Agroalimentarios, Economía y Empresa, Gestión Comercial y Valoración de la Empresa Agroalimentaria. En la siguiente tabla se identifican las titulaciones, cursos y asignaturas donde se ha aplicado la metodología. Estas asignaturas las podemos dividir en dos grandes bloques. Las dos primeras se centran en conceptos de Investigación de mercados mientras que las tres últimas se centrarán en conceptos de Economía de la empresa.

Tabla 1: Titulaciones, cursos y asignaturas

\begin{tabular}{lll}
\hline Titulación & Curso & Asignatura \\
\hline $\begin{array}{l}\text { Doble Grado en Derecho y Administración y } \\
\text { Dirección de Empresas }\end{array}$ & $4^{\circ}$ & Investigación de Mercados \\
\hline
\end{tabular}




\begin{tabular}{lcl}
\hline $\begin{array}{l}\text { Máster de Ingeniería Agronómica } \\
\text { Grado en Ingeniería Agronómica y del Medio } \\
\text { Rural }\end{array}$ & $1^{\circ}$ & $\begin{array}{l}\text { Investigación de Mercados Agroalimentarios } \\
\text { Economía y Empresa }\end{array}$ \\
$\begin{array}{l}\text { Grado en Ingeniería Agronómica y del Medio } \\
\text { Rural }\end{array}$ & $2^{\circ}$ & $\begin{array}{l}\text { Gestión Comercial y Valoración de la } \\
\text { Empresa Agroalimentaria }\end{array}$ \\
\hline
\end{tabular}

\section{OBJETIVOS}

La finalidad general de este proyecto radica en promover la innovación docente en la Enseñanza Superior utilizando para ello las TIC, creando los recursos y materiales necesarios para poder desarrollar una experiencia de Flipped Classroom y TIC con la finalidad de satisfacer los siguientes objetivos:

1. Mejorar la motivación y asistencia a clase.

2. Conocimiento de estrategias e instrumentos de búsqueda de información en internet.

3. Fomentar la evaluación formativa y la discusión y participación en clase.

4. Mejorar los resultados del proceso de aprendizaje de los alumnos.

5. Mejorar la capacidad de razonar de los alumnos en la aplicación de los conceptos teóricos para la resolución de problemas.

6. Fomentar el trabajo colaborativo.

7. Fomentar el pensamiento creativo y reflexivo.

8. Evaluar la posible mejora de la calidad docente y aprendizaje del alumno a través de un análisis estadístico del desempeño de la herramienta.

\section{MATERIAL Y MÉTODOS}

\subsection{Metodología}

La metodología y actividades necesarias para la consecución de los objetivos descritos anteriormente se fundamentó principalmente en virtualizar algunas sesiones prácticas y teóricas. Asimismo, y considerando el carácter eminentemente práctico de algunas de las asignaturas se dotó al alumnado de bases de datos externas para que pudiesen aplicar los conocimientos adquiridos en clase junto con los videos emplados para la "Flipped Classroom". Para imbuir al alumnado con un espíritu de curiosidad por descubrir, se favoreció el trabajo en grupo en las prácticas en aula de informática, así como se informó que el contenido generado en el aula se emplearía en la evaluación final de la asignatura.

Entre las actividades relativas a la metodología para la consecución de los objetivos descritos anteriormente se ejecutaron las siguientes (en algunas de estas actividades se intentó que el material fuese en inglés para que el alumnado interiorizase la necesidad de formarse en dicho idioma):

Búsqueda, adaptación y diseño de recursos que el alumno pueda visualizar fuera del aula. Así se pusieron a disposición del alumnado entre otros aspectos:

Videos docentes de profesorado de otras universidades sobre métodos cuantitativos para la Investigación de Mercados y para Economía de la Empresa abordados en las asignaturas:

- Regresión logística: https://goo.gl/mRHm7v; https://goo.gl/NLYcfZ

- Análisis cluster MIT: https://goo.gl/sAZzxo

- Método Canvas para modelos de negocio: https://www.youtube.com/watch?v=i1Le5GYkBT8

- Cálculo de amortización de préstamos: https://www.youtube.com/watch?v=heum3Q3O_Yw 
Materiales de consulta de diversas fuentes en relación con conceptos estadísticos clave para abordar una Investigación de Mercados y la financiación a largo plazo en Economía de la Empresa

- http://wpd.ugr.es/ bioestad/guia-spss/practica-8/

- http://www.bioestadistica.uma.es/baron/apuntes/

- https://www.uoc.edu/in3/emath/

- Relación entre TIN, TIE, TAE, TIR, TRE: http://yirepa.es/tin-tie-tae-tir-tre.html

- Tabla amortización : http://www.tabla-amortizacion.es/

Enlaces a repositorios de Trabajos fin de Grado para que el alumno interiorizase la potencialidad de la disciplina Investigación de Mercados como una herramienta básica para la investigación en el mundo empresarial y académico y la amortización de préstamos como proceso fundamental para la viabilidad de inversiones.

- https://goo.gl/uaY4Ak

- $\quad$ https://goo.gl/mMXVME; https://goo.gl/3t2frZ; https://goo.gl/sDWZQM

Conjuntamente, se procedió a la grabación y edición de videos para el desarrollo de la experiencia "Flipped Classroom" y puesta en valor de material anterior en las asignaturas de Investigación de Mercados. Para ello, la adaptación de algunos de los contenidos de la asignatura se realizó con Camtasia, software especializado de captura, grabación y edición de video. A modo de ejemplo, en el siguiente link se puede encontrar una clase práctica en SPSS sobre el cumplimiento de supuestos en un modelo de regresión lineal: https://goo.gl/3v3LRM. En algunas unidades temáticas, y con la intención de enriquecer la experiencia "Flipped Classroom", se proveyó al alumnado, además de con el video de su profesor, con otro adicional de otro docente de una universidad extranjera (particularmente dicha estrategia se empleó en el tema relativo al análisis cluster empleando el video, citando anteriormente, perteneciente al MIT y su estrategia "OpenCourseWare: https://ocw.mit.edu/index.htm). Adicionalmente, se dotaba al alumno con bases de datos reales para llevar a cabo una implementación práctica del conocimiento adquirido (a modo de ejemplo : https://goo.gl/jxhtyG). Asimismo, la asignatura dispone, como material docente común para todos los alumnos, de un manual teórico y de prácticas editado por el profesorado de la misma.

El objetivo de todas las fuentes ofrecidas ha sido impulsar al alumnado a realizar actividades complementarias que retroalimenten el nuevo paradigma de la "Flipped Classroom" e incentivar que interiorizen el concepto de la "Flipped Classroom" a través de su participación en dinámicas de grupos en las clases prácticas. Concretamente, para el grupo experimental seleccionado se creo una comunidad en Moodle para indicar al alumnado participante que para la siguiente clase debían traer trabajado el material docente descrito anteriormente. Debido a la novedad de dicho enfoque, se seleccionó como grupo experimental aquel que permitía un mejor ajuste temporal. Por tanto, si la clase presencial común para el gran grupo acababa el martes, se seleccionó como grupo experimental un grupo de prácticas de los viernes, para que dispusiesen de tiempo suficiente. Así, durante la clase práctica de los viernes, en lugar de realizar una aplicación práctica de la teoría donde el alumno va replicando en su ordenador lo que el profesor va explicando, se procedió a realizar una puesta en común inicial sobre las principales dificultades encontradas. Dichas dificultades se plasmaban esquematicamente por el docente en la pizarra y a continuación se procedía a utilizar una dinámica brainstorming (tormenta de ideas) para identificar por parte del profesor si alguno de los compañeros podían dar solución a las dificultades identificadas. En caso positivo, se procedía a dar voz al alumnado para que ejerciesen el rol docente explicando a sus compañeros la solución identificada. En función de la respuesta, el profesor corregía, matizaba o profundizaban en la explicación ofrecida por el alumno.

Con la finalidad de buscar retroalimentación por parte del alumnado, la gran mayoría de los contenidos generados fueron implementados en una estrategia de gamificación empleando el software libre Kahoot gracias a las posibilidades del BYOD (Bring Your Own Device), ya que la inmensa mayoría de los alumnos tiene un dispositivo movil que les permite el uso de este software interactivo. Por tanto, a partir de todas aquellas dudas que surgían en clase se pedía a los alumnos que confeccionase una pregunta de examen que ellos formularían si fuesen docentes. Posteriormente, dichas preguntas eran modificadas por el docente y pasaban a formar parte de 
una banco de preguntas. Esta estrategia de gamificación persiguió difundir en los alumnos un rol activo en un proceso de mejora continua donde ellos mismos y con su propio contenido se autoevalúan. A modo de ejemplo, en el siguiente link se puede encontrar algunas de estas actividades: https://goo.gl/3BbB6V.

\subsection{ANÁLISIS DE LA METODOLOGÍA}

La estadística ha sido empleada para el análisis del desempeño de la nueva metodología respecto a un enfoque tradicional. Con la finalidad de evaluar cuantitativamente la experiencia piloto se implementó en uno de los cuatro grupos de prácticas de la asignatura de Investigación de mercados del Doble Grado en Derecho y Administración y Dirección de Empresas. Como los integrantes de los grupos se asignaron aleatoriamente no debería existir ninguna razón a priori por la que el estudio pudiese ester sesgado por algún factor externo no controlado. No obstante, se produjeron cambios en la asignación aleatoria inicial debido a incompatibilidad horaria cuyo impacto sobre el experimento es indeterminado.

Para la evaluación de la experiencia implementada se generó una dinámica de grupo final. Esta dinámica consistió en que los alumnos expusieran las ventajas o dificultades que les ofrecía el método empleado para poder evaluar el nivel de satisfacción. Por otra parte, se comparó el desempeño académico de los alumnos de este grupo experimental respecto al resto (metodología tradicional). Para ello, se realizó una comparación de las calificaciones obtenidas, en el examen de evaluación, para aquellas partes del mismo ejecutadas con la metodología "Flipped Classroom" respecto a la tradicional.

\section{RESULTADOS OBTENIDOS Y DISCUSIÓN}

La propuesta educativa pretendía mejorar algunas de las carencias generalmente detectadas en los alumnos, especialmente en los primeros años de carrera universitaria, como son la baja motivación, la actitud pasiva, el absentismo y abandono, la baja utilización de tutorías y el bajo nivel de información sobre la realidad profesional y empresarial.

Respecto a las asignaturas que se imparten en el primer año de grado al final del curso, buena parte del alumnado llega desmotivado y no asiste a clase. Por otro lado, en las asignaturas pertenecientes a cursos superiores el principal problema es la falta de una comprensión profunda de la materia para que el alumando sea capaz de aplicar los conceptos y herramientas a la solución de distintos problemas.

Los resultados del análisis estadístico sobre el desempeño académico de los alumnos expuestos a la metodología "Flipped Classroom" no arrojaron diferencias estadísticamente significativas. Concretamente, como se puede observar en la prueba t de comparación de medias, a pesar de que la nota media del grupo experimental expuesto a la metodología "Flipped Classroom" fue superior (61,82 sobre 100) a la del grupo no expuesto (58,84 sobre 100), no se puede afirmar que la nota media de los expuestos sea estadísticamente diferente a las del grupo control.

No obstante, para evaluar estadísticamente ambas metodologías de forma certera se debería haber realizado una asignación aleatoria de los sujetos al grupo experimental y al control. Si bien al comienzo se logró dicha característica de aleatoriedad, al final no se pudo garantizar dicha característica deseable de aleatoriedad debido a cambios de los alumnos en los grupos por incompatibilidad horaria. No obstante, nada hace pensar que tal efecto de cambios en los grupos esté relacionado a priori con algún sesgo de autoselección muestral que pudise estar sesgando los resultados.

Tabla 1. Estadísticas de grupo 


\begin{tabular}{lccccc}
\hline & $\begin{array}{c}\text { Grupo } \\
\text { Experimental }\end{array}$ & N & Media & $\begin{array}{c}\text { Desviación } \\
\text { estándar }\end{array}$ & $\begin{array}{c}\text { Media de error } \\
\text { estándar }\end{array}$ \\
\hline \multirow{2}{*}{ Calificación } & 1.00 & 23 & 61.8261 & 20.96675 & 4.37187 \\
& 0.00 & 59 & 58.8475 & 18.64719 & 2.42766 \\
\hline
\end{tabular}

Tabla 2. Estadísticas de grupo. Prueba de Levene y prueba t

\begin{tabular}{llcccc}
\hline & & \multicolumn{2}{c}{$\begin{array}{c}\text { Prueba de Levene de } \\
\text { calidad de varianzas }\end{array}$} & \multicolumn{2}{c}{$\begin{array}{c}\text { prueba t para la } \\
\text { igualdad de medias }\end{array}$} \\
& Se asumen varianzas iguales & $\mathrm{F}$ & $\mathrm{Sig}$ & $\mathrm{t}$ & $\mathrm{gl}$ \\
\cline { 3 - 6 } Calificación & .702 & .405 & .627 & 80 \\
& No se asumen varianzas iguales & & & .596 & 36.348 \\
\hline
\end{tabular}

Tabla 3. Prueba de muestras independientes

\begin{tabular}{|c|c|c|c|c|}
\hline & & \multicolumn{3}{|c|}{ prueba $t$ para la igualdad de medias } \\
\hline & & $\begin{array}{c}\text { Sig. } \\
\text { (bilateral) }\end{array}$ & $\begin{array}{l}\text { Diferencia de } \\
\text { medias }\end{array}$ & $\begin{array}{l}\text { Diferencia de } \\
\text { error estándar }\end{array}$ \\
\hline \multirow{2}{*}{ Calificación } & Se asumen varianzas iguales & .532 & 2.97863 & 4.74749 \\
\hline & No se asumen varianzas iguales & .555 & 2.97863 & 5.00068 \\
\hline
\end{tabular}

Tabla 4. Prueba de muestras independientes

\begin{tabular}{llcc}
\hline & & \multicolumn{2}{c}{$\begin{array}{c}\text { prueba t para la igualdad de medias } \\
95 \% \text { de intervalo de confianza de la diferencia }\end{array}$} \\
\cline { 3 - 3 } Calificación & Se asumen varianzas iguales & Inferior & Superior \\
\hline & No se asumen varianzas iguales & -6.46917 & 12.42643 \\
\hline
\end{tabular}

Finalmente, creemos que el proyecto de innovación docente ha conseguido alcanzar los objetivos planteados. En concreto, podemos decir que la metodología utilizada ha permitido mejorar:

1. La motivación de los alumnos al participar de manera activa en el proceso de aprendizaje utilizando herramientas TIC cercana a los alumnos.

2. La responsabilidad en el trabajo en grupo realizado en clase.

3. La evaluación formativa al permitir valorar los progresos del aprendizaje mediante un proceso de retroalimentación o feedback.

4. Los conocimientos y la imagen del mundo empresarial.

5. La valoración de las propias capacidades para el emprendimiento.

Todo ello ha contribuido a mejorar el aprendizaje de la asignaturas. Sin embargo, en la aplicación de la experiencia Flipped Classroom se detectaron varias dificultades que vale la pena comentarlas. Si bien la innovación metodológica es bien acogida por los alumnos, del focus group realizado con ellos sobre las principales limitaciones de la misma, se desprende lo siguiente: 
1. El visionado previo del material docente en casa requiere de un gran compromiso por parte del alumnado que muchas veces no está dispuesto a asumir. En este sentido, si se pretende implementar un enfoque holístico de "Flipped Classroom" sería necesario internalizar la metodología de forma completa en la guía docente.

2. La duración de los videos no debe sobrepasar los 15 o 20 minutos. Según la propia experiencia realizada en clase, el visionado de videos más allá de los 20 minutos es muy complicado. Por tanto, se requiere de un troceado previo de las lecciones para adecuarlo a los tiempos de fatiga estimados.

3. No obstante, el rol del profesor es difícilmente sustituible por la metodología Flipped Classroom ya que los comentarios generalizados por lo estudiantes fueron: "profesor, con usted en vivo y en directo me entero mejor"; "profesor usted transmite mejor el conocimiento interactuando con nosotros directamente"; "los videos son mucho más aburridos que una clase donde usted interactúan con nosotros", etc.

No obstante, al mismo tiempo llegaron al consenso de que si estuviesen todos los materiales disponibles en esta versión de "Flipped Classroom" sería muy útil siempre y cuando el rol activo del profesorado en la clase presencial sobresalga. Es decir, si bien valoran muy positivamente la experiencia indicaron que dicha metodología debería reservarse para las partes más complicadas de la asignatura. De este modo, el número de videos y la duración de los mismos sería asumible por los estudiantes. Por otra parte, también indicaron que es una muy buena herramienta para aquellos alumnos que les gusta ir repasando o para resolver dudas de última hora antes de la evaluación.

Respecto a los materiales complementarios como la consulta de diferentes webs temáticas junto con la entrega de casos prácticos asociados a las sesiones flipped classroom no fue del todo satisfactorio. Con respecto a esta parte en concreto de la experiencia, se optó por ser una actividad de naturaleza voluntaria, por lo que la implicación por parte del alumnado fue menor que respecto al visionado de los videos. En relación con lo anterior, cualquier innovación metodológica que se pretenda implantar satisfactoriamente, necesariamente debe incluirse en la guía docente y evaluarla en función de su importancia en la nota final. Como en cualquier otro ámbito debe existir siempre un incentivo, independientementemente de su cuantía, para embarcar al alumno en un cambio de paradigma de aprendizaje.

Respecto a las actividades de gamificación en el aula fueron muy bien acogidas por el alumnado y complementan de forma muy adecuada la dinámica Flipped Classroom. En el caso concreto de las asignaturas de economía, al tener el examen partes muy diferenciadas como el tipo test, la resolución de problemas y la interpretación de modelos multivariantes, el alumnado encontró muy pertinente la actividad de gamificación para ir asentando conceptos progresivamente. Asimismo, el alumnado encuentra mucho más conveniente dichas actividades que los test con autoevaluación suministrados en Moodle. La principal desventaja es que su uso continuado puede poner en peligro terminar el temario de forma completa, por tanto, se recomienda su uso en las partes más relevantes del mismo.

\section{CONCLUSIONES}

El proyecto de innovación ha permitido claramente innovar en la docencia de las asignaturas propuestas. En este sentido, cabe destacar que la metodologúa Flipped Classroom mejora el feedback que el profesor recibe de los alumnos en tiempo real, permitiendo adaptarse al ritmo del aprendizaje de los alumnos. Esta experiencia ha demostrado ser muy valiosa para incrementar las posibilidades del alumnado en la adquisición de conocimiento. No obstante, el propio alumnado reconoce que no puede entenderse como una estrategia de sustitución del profesor porque la trasmisión de conocimiento no se realiza igual de bien. Por tanto, la principal área crítica de mejora respecto a la Flipped Classroom es que el alumnado se conciencie de la necesidad de estudiar y visionar los videos y los materiales de apoyo por adelantado para que cuando se llegue a la clase se pueda destinar a la resolución de dudas y a la realización de supuesto prácticos reales. Asimismo, la duración de los videos no debería sobrepasar los 15 o 20 minutos como límite superior puesto que su utilidad se ha 
demostrado muy reducida a partir de dicho nivel. Considerando todas estas unidades de mejora, se podría explotar toda la potencialidad de un enfoque Flipped Classroom.

Finalmente, la implementación práctica del engfoque Flipped Classroom requiere necesariamente de una plasmación tácita en la guía docente para tener el poder vinculante necesario para que el estudiante haga el esfuerzo de revisar en casa el soporte audiovisual temático y los materiales de apoyo.

Sin embargo, quedan aún cuestiones mejorables y que animan a seguir avanzando en el campo de la innovación docente. Así, creemos que es necesario incorporar mejoras en la evaluación del aprendizaje, de manera que los alumnos sean más partícipes de la evaluación del trabajo realizado. Tanto el entorno de trabajo en red en la nube creado con este proyecto de innovación como las nuevas herramientas moodle disponibles en el Aula Virtual de la UCO permiten procesos de co-evaluación que creemos necesario desarrollar. Por otro lado, también se requiere introducir un sistema de evaluación de la metodología docente utilizada que permita cuantificar de algún modo el cumplimiento de los objetivos propuestos. Es decir, el proyecto de innovación realizado requiere someterse a un proceso de validación más objetivo que permita confirmar la calidad del mismo y detecte los aspectos que puedan ser mejorables.

\section{AgRADECIMIENTOS}

Agradecemos las sugerencias y el apoyo de nuestros colegas del grupo de investigación WEARE (www.uco.es/weare) así como las aportaciones de los revisores.

\section{BIBLIOGRAFÍA}

ABEYSEKERA, L. y DAwsOn, P. "Motivation and cognitive load in the Flipped classroom: definition, rationale and a call for research", Higher Education Research \& Development 2015/34, p. 1-14.

ALBERT, M. Y BEATTY, B.J., “ Flipping the classroom applications to curriculum redesign for an introduction to management course: Impact on grades”, Journal of Education for Business 2015/89, p. 419-424.

Bergmann, J. y SAms, A. Flip Your Classroom: Talk to Every Student in Every Class Every Day. EEUU, 2012.

Bishop, J. L. Y VerLeger M. A., "The Flipped Classroom: A Survey of the Research", 120th ASEE Annual Conference \& Exposition, Atlanta, 2013.

Estevez, J., Garcia-Marin, A.P. y Ayuso-MuÑOZ, J.L. "Self-Perceived Benefits of Cooperative and ProjectBased Learning Strategies in the Acquisition of Project Management Skills", International Journal of Engineering Education 2018/34, p. 1-11.

Good, T. L. Y LAVIGNE, A. L. Looking in classroom. New York, 2017.

O'FlAHERTY, J. y PhilLiPS, C. "The use of flipped classrooms in higher education: A scoping review", The internet and bigher education 2015/25. p. 85-95. 